\section{How do characters evolve?}

Arising from: R. E. Ricklefs Nature 430, 338-341 (2004)

Rim icklefs ${ }^{1}$ claims to show that morphological evolution in birds is associated with speciation events - that is, it is punctuational - by inference from data on only species number, clade age and character variance from a range of passerine clades. $\mathrm{He}$ suggests that variance increases in proportion with clade age under gradual change, but in proportion to the logarithm of species number if change is punctuational. Here I show that both clade age and the logarithm of species number independently predict variance under both gradual and punctuational change, rendering Ricklefs' results uninformative about his central hypothesis.

First, I simulated 100 clades that survived to the same age (60 units) under a constantrates birth-death process (speciation rate, 0.2 ; extinction rate, 0.16 ). Trees contained from one species (excluded from analyses) to 238 species (mean, 49.3). I evolved two characters on each tree under brownian motion - one gradual, the other changing only at speciation. $\log$ (species number) is a highly significant predictor of variance in the evolved trait under both gradual $\left(t_{96}=5.42\right.$, $\left.P<0.0001, r^{2}=0.23\right)$ and punctuational change $\left(t_{96}=7.57, P<0.0001, r^{2}=0.37\right)$, even among clades of the same age.

Second, I evolved 100 surviving clades until they reached a fixed size (50 extant species) by using the same process and evolved characters as before. Clade age ranged from 23.7 to 176.2 time units and significantly (though weakly) predicted variance in the evolved trait under both gradual $\left(t_{98}=2.03, P=0.046, r^{2}=0.03\right)$ and punctuational $\left(t_{98}=2.32, P=0.02, r^{2}=0.04\right)$ models, even among clades of the same size.

These results are not surprising. Under gradual change, variance accumulates along phylogenetic branches ${ }^{2}$. Larger clades have more total branch length within them, even in same-aged clades (across my trees, Spearman's $r=0.98$ ), and so have more variance in gradually evolved traits. With punctuational change, variance accumulates only at speciations, but older clades have experienced more speciation (and extinction) events even in same-sized clades - to an extent that depends on the extinction rate ${ }^{3}$. Clade age is therefore a good predictor of number of speciations across the same-sized trees (Spearman's $r=0.81$ ).

Ricklefs ${ }^{1}$ found that $\log$ (species number) but not clade age independently predicted morphological variance in multiple regressions, and concludes from this that morphological evolution in birds seems to be associated with cladogenesis. However, my simulations show that his significant results for $\log$ (species number) are expected under both models. Further, both models predict that clade age too will correlate with variance, but Ricklefs found no significant association. Why not? Ricklefs proposes a model in which speciation promotes gradual divergent change. It is unclear what predictions his model makes about his data. Alternatively, the negative result may simply be a type II error. Clade age was only weakly predictive of variance in my simulations, and the phylogeny from which the clade ages are taken ${ }^{4}$ conflicts markedly with more recent evidence ${ }^{5}$.

The mode of character evolution can sometimes be inferred using detailed phylogenetic information ${ }^{3,6,7}$. Clade ages and species numbers alone are not enough.

\section{Andy Purvis}

Department of Biological Sciences,

Imperial College London, Silwood Park Campus,

Ascot, Berks SL5 7PY, UK

e-mail:a.purvis@imperial.ac.uk

doi:10.1038/nature03092

1. Ricklefs, R. E. Nature 430, 338-341 (2004).

2. Felsenstein, J. Am. Nat. 125, 1-15 (1985).

3. Bokma, F. J. Evol. Biol. 15, 1048-1056 (2002).

4. Sibley, C. G. \& Ahlquist, J. E. Phylogeny and Classification of Birds: A Case Study of Molecular Evolution (Yale Univ. Press, New Haven, Connecticut, 1990).

5. Barker, F. K., Cibois, A., Schikler, P., Feinstein, J. \& Cracraft, J. Proc. Natl Acad. Sci. USA 101, 11040-11045 (2004).

6. Mooers, A. Ø., Vamosi, S. M. \& Schluter, D. Am. Nat. 154, 249-259 (1999)

7. Pagel, M. Nature 401, 877-884 (1999).

Ricklefs replies - Purvis ${ }^{1}$ states that, under random gradual change, clades accumulate variance in proportion to their total branch length. Accordingly, at a given age, clades with more species should exhibit greater variance. This is incorrect, as shown both analytically ${ }^{2,3}$ and by simulation ${ }^{4,5}$, and the error underlines a misunderstanding of my analysis.

Variance depends strictly on the average time of divergence between species within a clade and not on the number of species or total branch length. The positive relationship between species number and variance in Purvis's simulations with fixed time reflects the occurrence of earlier branch points in what turn out to be larger clades. Had Purvis simulated gradual evolution in an unconstrained speciation-extinction process with varied clade ages, he would have obtained a significant partial correlation between variance and time, which I was unable to detect in clades of passerine birds. The weaker time effect in Purvis's second simulation with constrained clade size reflects the distribution of most nodes in each tree at similar depth, regardless of the age of the root.

To determine whether time itself, and hence gradual evolution, contributes to trait variance, one must analyse data, whether observed or simulated, in which time and the number of species are allowed to vary independently. Purvis's simulations lack this independence: the first does not vary clade age; the second holds the species number constant. The results are not surprising, and they do not address the validity of my analysis. Although average divergence time and number of species are correlated among clades, multiple regression allows one to identify unique contributions of time and species number to trait variance among clades of different ages.

Purvis's comment about a type II error (failing to detect a true relationship) is of more concern, particularly because relative age, based on DNA-hybridization phylogeny $^{6}$, is estimated less well than the number of species. In a test of the monophyly of 40 out of 106 (from ref. 6) tribe-to-family-level clades using a maximum-likelihood analysis of over 4 kilobases of the RAG-1 and RAG-2 genes $^{7}$, only three were found that were significantly paraphyletic compared with 27 strongly supported and 10 ambiguous clades ${ }^{8}$. This does not "conflict markedly" with the monophyly of clades used in my analysis. It is of more importance, however, that the relative ages of clades in the sequence-based and DNA-hybridization phylogenies were not compared.

If the phylogeny in ref. 6 provides a reasonably accurate view of clade age, then the absence of a significant time effect on variance among different-aged clades would be sufficient to reject a model of gradual evolution that is independent of species number. Gradual evolutionary divergence, whether fast or slow, driven by interactions among species in a clade (as opposed to punctuated evolution associated with speciation ${ }^{9}$ ) is also species-dependent, rather than time-dependent, inasmuch as the pressure to diversify is in some way proportional to species number. Increasing knowledge of phylogenetic relationships makes this an opportune time to examine more closely the generation of trait variance in diversifying clades.

\section{Robert E. Ricklefs}

Department of Biology, University of Missouri

at St Louis, St Louis, Missouri 63121-4499, USA

e-mail: ricklefs@umsl.edu

doi:10.1038/nature03093

\footnotetext{
1. Purvis, A. Nature doi:10.1038/nature03092 (2004).

2. Slatkin, M. Paleobiology 7, 421-425 (1981).

3. Gavrilets, S. Proc. R. Soc. Lond. B 266, 817-824 (1999).

4. Raup, D. M. \& Gould, S. J. Syst. Zool. 23, 305-322 (1974).

5. Valentine, J. W., Collins, A. G. \& Meyer, C. P. Paleobiology 20 , 131-142 (1994)

6. Sibley, C. G. \& Ahlquist, J. E. Phylogeny and Classification of the Birds of the World (Yale Univ. Press, New Haven, Connecticut, 1990).

7. Sibley, C. G. \& Monroe, B. L. Jr Distribution and Taxonomy of Birds of the World (Yale Univ. Press, New Haven, Connecticut, 1990).

8. Barker, F. K., Cibois, A., Schikler, P., Feinstein, J. \& Cracraft, J. Proc. Natl Acad. Sci. USA 101, 11040-11045 (2004).

9. Gould, S. J. \& Eldredge, N. Paleobiology 3, 115-151 (1977).
} 